\title{
Review of: "B.P.F.C PURE GROWTH FACTOR, PDT AND LLLT FOR THE TREATMENT OF SEVERE MRONJ NON- RESPONSIVE TO PROLONGED ANTIBIOTIC THERAPY"
}

\author{
Funda Goker ${ }^{1}$ \\ 1 University of Milan
}

Potential competing interests: The author(s) declared that no potential competing interests exist.

- The manuscript has value and would attract the readers of the Qeios. The topic is certainly interesting and has scientific value in the treatment of severe MRONJ patients. This is a case report on utilization of new platelet-rich plasma preparation, Photodynamic therapy and, Low level laser therapy for the treatment of severe MRONJ. The article needs few minor revisions.

- ABSTRACT: Abbreviations are not usually written in the abstracts, so they can be erased but can be kept in the introduction when they are first mentioned.

- English language needs some corrections.

For example: she received a surgery extraction of the 3.7 and she did not heal....

The tooth \#37 was extracted and the surgical site did not heal within eight weeks...

- Diagnosis of MRONJ can be explained in more details. No healing within 8 weeks can be highlighted for the diagnosis of MRONJ. It is not clear if there was already an osteonecrotic lesion in \#36-37 and the teeth was extracted due to that lesion or the lesion occured after the extraction

- Instead of using definitive terms like "This treatment is able to provide perfect healing with the integrity of the hard and soft tissue after six months."

Can be written as: The treatment modality mentioned in this study seems to be beneficial, promising... etc 